\title{
Prospective management of end-stage renal failure within a conservative care programme
}

\author{
Neeraj Dhaun, Johanna Mackenzie, Jane Goddard, Caroline Whitworth
}

\begin{abstract}
Progression to end-stage renal disease (ESRD) is a major issue for heath care systems both clinically and financially. Given dialysis may not prolong life, and may indeed impair quality of life, alternative options for these patients such as conservative care are urgently needed. We appointed a dialysis charge nurse who had many years of experience of working with patients on dialysis to spearhead the newly set up Conservative Care Programme (CCP) in the Edinburgh Renal Unit. The rationale was to work as part of the renal multidisciplinary team to support patients and their families to make an informed shared decision whether to opt for dialysis or to follow the CCP.

From the perspective of the patients, their families and carers we have received positive feedback since starting the CCP - thank you cards; phone calls both to the CCP Nurse Specialist and the renal unit affirming the positive experience patients had during the conservative management of their renal failure. Whilst continuing to provide the best quality of care to renal patients in NHS Lothian and Borders, the number of prevalent dialysis patients in our catchment area has fallen significantly over the last few years as demonstrated by Scottish Renal Registry data. These benefits are potentially applicable to other renal units across the UK.
\end{abstract}

\section{Problem}

Progression to end-stage renal disease (ESRD) is a major issue for heath care systems both clinically and financially. There are currently over a million patients worldwide on dialysis, with the number increasing by $\sim 7 \%$ yearly, particularly in the elderly, many of whom have significant extra-renal comorbidities and will do poorly on dialysis. [1] Furthermore, dialysis is expensive costing $\sim £ 30,000$ per patient per year in the UK. [2] Given dialysis may not prolong life and may indeed impair quality of life alternative options for these patients such as conservative care are urgently needed.

\section{Background}

Chronic kidney disease (CKD) is a major health problem affecting $10-16 \%$ of the world's general adult population. [3-8] Progression to ESRD remains a major clinical problem and there are currently over a million patients worldwide on dialysis, with the number increasing by $\sim 7 \%$ yearly, particularly in the elderly, many of whom are dependent and frail. [1] Furthermore, survival once on dialysis is poor.

The benefits of dialysis for elderly, dependent patients with ESRD have been questioned. The prospects for rehabilitation in such patents tend to be slim, and prognosis is often poor. [9, 10] Dialysis in such circumstances can pose huge additional burdens for patients and their carers. In recent years, the concept of the "Conservative Care Programme" (CCP) has gained sway in an attempt to provide a comprehensive package of care to patients who have chosen to forego dialysis. Conservative management of ESRD involves a shift from efforts to prolong life to those that focus on care, quality of life and symptom control.

\section{Baseline Measurement}

Our assessment of the scale of the problem was qualitative. Many of us have many years of experience working as part of a multidisciplinary team looking after patients on dialysis. We have all witnessed first-hand how difficult dialysis could be for the elderly and their families and were thus moved to instigate a CCP in our unit.

\section{Design}

Intervention: appointment of a Conservative Care Programme (CCP) Nurse Specialist

We appointed a dialysis charge nurse who had many years of experience of working with patients on dialysis to spearhead the newly set up CCP in Edinburgh. The rationale was to work as part of the renal multidisciplinary team to support patients and their families to make an informed shared decision whether to opt for dialysis or to follow the CCP.

The CCP targets the active treatment of symptomatic anaemia, fluid overload, blood pressure control and management of uraemic symptoms to promote the best quality of life. The programme allows these patients to receive the best quality of care but without having to attend the hospital on a regular basis to receive this care. The CCPI also aims to provide bereavement counselling for families and carers.

Challenges to setting up a CCP

The CCP nurse specialist is the key figure involved in setting up, and in the success of, a CCP. This individual should have extensive 
experience of working with patients with advanced renal failure and those on dialysis but also possess the key qualities essential for the $\mathrm{CCP}$, namely excellent communication skills, the ability to liaise with other health care professionals in both primary and secondary care, and the ability to work as part of a multi-disciplinary team.

The engagement of other members of the renal unit (especially the medical team) in the CCP is important. Patients with CKD can only choose conservative care as the management of their ESRD if this is an option offered by their nephrologist. In order to engage others, the CCP nurse specialist needs to have a positive attitude to conservative care. To raise the profile of the CCP we have had positive feedback from patients and their families directly to their nephrologist showing them directly the utility of this programme. We have also provided in-house education to all members of the renal team as well as education days to GPs and health care professionals in primary care to raise awareness of the CCP.

\section{Strategy}

Given the qualitative parameters of the role of the CCP Nurse Specialist the strategy for improvement was experience based with each patient (and their family) encounter informed by those preceding it. The role of the CCP Nurse Specialist has developed from solely being involved in hospital care to working in partnership with the multidisciplinary team both in primary and secondary care. In primary care, they interface directly with the patient, their carers and family and provide education and support. They also discuss the patient's care with their GP, district nurses, and community palliative care team, with an emphasis on advance care planning. When the patient is in hospital they are involved in discussions with the medical team, occupational therapists, physiotherapists, social workers, nursing staff, palliative care team and the members of the spiritual care team. At the centre of these discussions is the patient along with their family and other carers. The CCP Nurse Specialist supports patients "opting out" of dialysis initiation as well as those withdrawing from dialysis. Importantly, they provide a strong communication link between the primary and secondary care givers as well as providing a voice for the patient and their families through their independent reviews. The focus of their discussion is centred around the importance of symptom control and quality over quantity of life.

\section{Results}

\section{Qualitative Results}

From the perspective of the patients, their families and carers we have received positive feedback since starting the CCP - thank you cards; phone calls both to the CCP Nurse Specialist and the renal unit affirming the positive experience patients had during the conservative management of their renal failure. We have also had money donated to the renal unit to support future work in this area.

Phrases used by families and carers of patients include:

"Professional, dedicated, source of friendship, moral support, help, understanding, kindness,

Loved one allow to die with dignity,

Patient's wishes met,

Good death,

A special person,

Wonderful support,

Thanks for keeping loved one as well as possible to the end"

\section{Quantitative Results}

Whilst continuing to provide the best quality of care to renal patients in NHS Lothian and Borders, the number of prevalent dialysis patients in our catchment area has fallen significantly over the last few years as demonstrated by Scottish Renal Registry (SRR) data (see Figure 1). According to the SRR reports the median age of incident patients starting dialysis in Lothian in 2004 was 64 years and this has fallen to 58 in 2012. Furthermore, the agestandardised incidence of new patients starting dialysis in 2007-2011 in NHS Lothian was 8.9 per 100,000 population, compared to $10.4 / 100,000$ for Scotland as a whole. At any one time we have 100 patients on the CCP casebook, $25 \%$ of whom have an estimated GFR $>10 \mathrm{ml} / \mathrm{min}$ so who would otherwise be on dialysis. In addition, the CCP Nurse Specialist is involved in the care of 3-5 patient deaths/month.

See supplementary file: ds1693.docx - "Figure 1"

\section{Lessons and Limitations}

Our experiences in NHS Lothian of a CCP are potentially more widely applicable to the UK. We plan to use the data and experiences accrued in our centre to improve health care provision for those patients not wishing to pursue dialysis as a treatment option for their ESRF. We will plan to do this in a number of ways:

1. Continued presentations at national renal meetings

2. Teaching sessions for nursing, medical and allied health professionals both locally and nationally - the latter through workshops at national meetings

3. Continued dialogue with the standard palliative care teams both locally and through presentations and workshops at national meetings - this will improve renal-specific knowledge for these health care professionals

4. Working towards anticipatory care planning for selected dialysis patients with a view to planning for a 'good death'

Given the increasing use of the CCP in Edinburgh, as well as the expanding role of the CCP nurse specialist, we have now employed another full-time CCP nurse specialist which allows for succession planning. 


\section{Conclusion}

Progression to ESRD is a major issue for heath care systems both clinically and financially. Given dialysis may not prolong life and indeed impair quality of life alternative options for these patients, such as conservative care, are urgently needed. The introduction of a CCP in our unit has not only improved patient care and quality of life but, whilst continuing to provide the best quality of care to renal patients in NHS Lothian and Borders, the number of prevalent dialysis patients in our catchment area has also fallen. These benefits are potentially applicable to other renal units across the UK.

\section{References}

1. Lysaght MJ. Maintenance dialysis population dynamics: current trends and long-term implications. J Am Soc Nephrol 2002; 13: S37-40.

2. http://www.kidney.org.uk/?campaigns/Transplantation/uktpress-dec03.html.

3. K/DOQI clinical practice guidelines for chronic kidney disease: evaluation, classification and stratification. Am J Kidney Dis 2002; 39: 1-266.

4. Chadban SJ, Briganti EM, Kerr PG, Dunstan DW, Welborn TA, Zimmet PZ, et al. Prevalence of kidney damage in Australian adults: The AusDiab kidney study. J Am Soc Nephrol 2003; 14:131-8.

5. Coresh J, Selvin E, Stevens LA, Manzi J, Kusek JW, Eggers $P$, et al. Prevalence of chronic kidney disease in the United States. JAMA 2007; 298: 2038-47.

6. Hallan SI, Coresh J, Astor BC, Asberg A, Powe NR, Romundstad S, et al. International comparison of the relationship of chronic kidney disease prevalence and ESRD risk. J Am Soc Nephrol 2006; 17: 2275-84.

7. Wen CP, Cheng TY, Tsai MK, Chang YC, Chan HT, Tsai $\mathrm{SP}$, et al. All-cause mortality attributable to chronic kidney disease: a prospective cohort study based on 462293 adults in Taiwan. Lancet. 2008; 371: 2173-82.

8. Levey AS, de Jong PE, Coresh J, El Nahas M, Astor BC, Matsushita $\mathrm{K}$, et al. The definition, classification, and prognosis of chronic kidney disease: a KDIGO Controversies Conference report. Kidney Int 2011; 80 : 17-28.

9. Chandna SM, Schulz J, Lawrence C, Greenwood RN, Farrington $\mathrm{K}$. Is there a rationale for rationing chronic dialysis? A hospital based cohort study of factors affecting survival and morbidity. BMJ. 1999; 318: 217-23.

10. Kurella Tamura M, Covinsky KE, Chertow GM, Yaffe K, Landefeld CS, McCulloch CE. Functional status of elderly adults before and after initiation of dialysis. New Engl J Med 2009; 361: 1539-47.

\section{Declaration of interests}

None of the authors have any declaration of interests. 